\title{
Thermal degradation kinetics and solid state, temperature dependent, electrical conductivity of charge-transfer complex of phenothiazine with chloranil and picric acid
}

\author{
M A ASHOK and B N ACHAR* \\ Department of Chemistry, University of Mysore, Mysore 570 006, India
}

MS received 22 August 2007; revised 11 October 2007

\begin{abstract}
Temperature dependent electrical conductivity and thermal degradation kinetics of charge-transfer (C-T) complexes of phenothiazine (PTZ) with p-chloranil (CHL) and picric acid (PA), are reported. These C-T complexes exhibited semiconducting behaviour. The activation energies for PTZ-CHL and PTZ-PA complexes are calculated based on their electrical conductivities measured over the temperature ranges $30-110^{\circ} \mathrm{C}$ and $30-90^{\circ} \mathrm{C}$, respectively. And these energies for PTZ-CHL and PTZ-PA are $0.54 \mathrm{eV}$ and $0.75 \mathrm{eV}$, respectively. The complexes are analysed for the kinetic parameters like the activation energy for decomposition and the Arrhenius pre-exponential factors in their pyrolysis region using Broido's, Coats-Redfern as well as Horowitz-Metzger methods. Using standard equations, thermodynamic parameters such as enthalpy, entropy and free energies, are calculated.
\end{abstract}

Keywords. Phenothiazine; charge-transfer complexes; electrical conductivity; thermal degradation.

\section{Introduction}

Phenothiazine derivatives belong to a big group of aromatic compounds. These derivatives are substituted in positions 2 and 10 and are commonly known as antipsychotic, anticholinergic and antihistaminic drugs. Due to their characteristic structure they exhibit valuable analytical properties (Kojlo et al 2001). They have been intensely studied in a number of fields such as chemical, biological and medical research owing to their pharmacological activities (Jones 1996; Nagy et al 1996; Tanaka et al 1997; Szocs and Seiler 2002; Ordway et al 2003; Mattana et al 2004). Phenothiazines are excellent electron donors as stated by Karremen et al (1959). The donor activity of phenothiazine is so high that even in the ground state there is practically the total transfer of electron to an acceptor resulting in the formation of charge-transfer $(\mathrm{C}-\mathrm{T})$ complexes (Karpinska et al 1996). Phenothiazine derivatives are much cheaper and could be easily substituted by different functional groups which will lead to electronic tuning of physical properties. Due to their structural effects they are expected to form a variety of charge-transfer complexes which exhibit interesting optical, electrical and magnetic properties (Singh and Singh 1997). Organic electronic materials provide a wide scope for researchers world wide for replacing conventional inorganic electronic materials (Gutmann and Lyons 1967; Torrance 1985). A

*Author for correspondence (bnachar@yahoo.com) few researchers have reported $\mathrm{C}-\mathrm{T}$ complexes of phenothiazine with various electron acceptors (Singh et al 1993; Singh and Singh 1996). p-Chloranil has been used effectively in spectrophotometry for the determination of various drugs by the formation of $\mathrm{C}-\mathrm{T}$ complexes (Gangopadhyay and Lahiri 2001; Salem 2002; Maher et al 2003; Darwish 2005; Sadeghi and Karimi 2006). Gutmann and Keyzer (1966) extensively studied the conductometric titrations of $\mathrm{C}-\mathrm{T}$ complexes in different solvent systems. A few researchers have reported the conductometric titrations of C-T complexes of chloranil with some donors (Dwivedi and Banga 1979; Srivastava et al 1982). Iida (1971) reported cation radical salts of phenothiazine and related compounds. He also reported the UV absorption spectra and magnetic susceptibility of phenothiazine and picric acid $\mathrm{C}-\mathrm{T}$ complexes. Shi et al (1988) reported the EPR study of $\mathrm{C}-\mathrm{T}$ complexes of ground state acceptor chloranil and a few donors. Achar and Krishnaswamy (1989) reported the ambient temperature electrical conductivity of $\mathrm{C}-\mathrm{T}$ complexes of phenothiazine with $p$-chloranil and picric acid. Basu and Choudhary (1992) reported C-T interactions in some phenothiazine drugs. Barigand et al (1970) reported the d.c. conductance of the solid complex obtained by melting stoichiometric amounts of phenothiazine and $p$ chloranil. However, a thorough literature survey revealed that temperature dependent electrical conductivity and thermal degradation studies of $\mathrm{C}-\mathrm{T}$ complex of phenothiazine with $p$-chloranil (PTZ-CHL) and picric acid (PTZ$\mathrm{PA}$ ) have not been done. In view of the fast growing field of organic electronics, the present investigation is 
expected to throw more light on the $\mathrm{C}-\mathrm{T}$ complexes of phenothiazine with $p$-chloranil and picric acid.

\section{Experimental}

Phenothiazine (Fluka, Germany) and $p$-chloranil (SigmaAldrich) were used as received. Picric acid (Qualigens Fine Chemicals, India) was recrystallized twice from doubly distilled water for constant melting point. For conductivity measurements, all the samples were compressed into pellets of $1.30 \mathrm{~cm}$ diameter and thicknesses ranging around $0 \cdot 1-0 \cdot 2 \mathrm{~cm}$ using Perkin-Elmer KBr Die under a pressure of $250 \mathrm{~kg} / \mathrm{cm}^{2}$. The TSI Techno search instruments, Thane, India, KBr press model-15 ton capacity was used for applying pressure. Conducting silver paint was coated on both flat surfaces of the pellets and electrical contacts with the electrodes were made by using the same paint. The resistance measurements were done using DOT-402 Digital Milli Ohm Meter and DOT-425 Insulation resistance tester, Bhandari Electronics and Electricals, Bangalore, India. Thermogravimetric analysis of PTZ-CHL and PTZ-PA complexes was performed in air atmosphere using TGA-7 Analyzer, Perkin-Elmer, USA, from ambient temperature to $750^{\circ} \mathrm{C}$ at a heating rate of $10^{\circ} \mathrm{C} / \mathrm{min}$. Elemental analysis for carbon, hydrogen, nitrogen and sulphur were done using Vario EL III CHNS analyzer, Germany. IR spectra were taken using JASCO FTIR-460 PLUS spectrophotometer, Japan.

\subsection{Preparation of phenothiazine-picric acid complex}

Phenothiazine $(0.9976 \mathrm{~g}, 0.05 \mathrm{M})$ and picric acid $(1.1468 \mathrm{~g}$, $0.05 \mathrm{M}$ ) in chloroform were mixed and stirred for half an hour in a beaker. A deep black coloured solution thus obtained was allowed to stand for 2-3 days in fuming cupboard. Black coloured long needle-type crystals were obtained. The crystals were separated by filtration and washed with carbon tetrachloride. The complex was recrystallized from chloroform and the crystals were dried in vacuum desiccator over phosphorous pentoxide.

\subsection{Preparation of phenothiazine-chloranil complex}

In the case of PTZ-CHL complex, phenothiazine $(0.9970 \mathrm{~g}$, $0.05 \mathrm{M})$ and $p$-chloranil $(1.2300 \mathrm{~g}, 0.05 \mathrm{M})$ in acetone were mixed and stirred for half an hour. The dark green complex formed was poured into a large petri dish and kept inside the fuming cupboard to evaporate the solvent. The green coloured solid obtained was powdered well and washed with carbon tetrachloride. The complex was dried in a vacuum desiccator over phosphorous pentoxide.

\section{Results and discussion}

PTZ-CHL and PTZ-PA are dark green and black in colour. Both the complexes are insoluble in water, but soluble in organic solvents like acetone, chloroform, acetonitrile etc. Elemental analysis is done for the first time to check the purity and stoichiometry of the complexes. The data supports $1: 1$ complex formation in both the cases. The molecular weight and formula are presented in table 1. The probable molecular structures are given (figures 1-2). FTIR data matched well with the earlier values reported by Achar and Krishnaswamy (1989) and is reported just for the sake of comparison (table 1). Thermogravimetric studies are done using analytical parameters as indicated in the experimental section. The maximum decomposition temperatures $\left(D T_{\max }\right)$ along with the other thermal decomposition kinetic parameters are presented in table 2. Literatures concerning the thermal decomposition of phenothiazine derivatives are rather poor. It is supposed that the thermal degradation in presence of air proceeds due to the oxidation of sulphur atom in the ring to sulphoxide, for the majority of these compounds (Bordea and Silberg 1964). The nature of TGA curves indicated that PTZ-CHL degraded in two steps whereas PTZPA degraded in three steps (figure 3). The major amount of degradation, 55\%, observed for PTZ-CHL is in the first step whereas it is observed at $50 \%$ in the second step for PTZ-PA. The second step degradation in the case of PTZ-PA complex is very small, to the extent of $10 \%$ and hence the thermodynamic parameters could not be calculated. At $600^{\circ} \mathrm{C}$, both the complexes degraded completely. The aim of the kinetic study of thermal analysis data is to find out the most probable kinetic model which best describes the process and allows the calculation of reliable values for the parameters like the order of reaction, activation energy, enthalpy of reaction, entropy of reaction, Gibb's free energy changes and the frequency factor. Many methods exist to characterize the degradation kinetics of various materials (Freeman and Carrol 1958; Friedman 1963; Horowitz and Metzger 1963; Coats and Redfern 1964; Ozawa 1965; Flynn and Wall 1966; Broido 1969; ASTM 1984; Agarwal and Sivasubramanian 1987). Three methods have been employed, Broido's, CoatsRedfern (C-R) and Horowitz-Metzger (H-M), for the evaluation of decomposition kinetics and the results obtained are compared and presented in table 1. In Broido's method, the thermal degradation process is considered to be of first-order and the calculations are done accordingly. In the case of $\mathrm{C}-\mathrm{R}$ and $\mathrm{H}-\mathrm{M}$ methods, the curve having the highest correlation coefficient values among the reactions of different orders are considered. The different equations employed to evaluate the degradation kinetics in three different methods are given below

Broido's method: $\quad \ln [-\ln y]=-\frac{E_{\mathrm{a}}}{R T}$.

Coats-Redfern method:

$\ln \left[\frac{-\ln y}{T^{2}}\right]=\ln \left[\frac{A R}{\beta E_{\mathrm{a}}}\left(1-\frac{2 R T}{E_{\mathrm{a}}}\right)\right]-\frac{E_{\mathrm{a}}}{R T} \quad$ for $n=1$, 
Table 1. Elemental and FTIR analytical data.

\begin{tabular}{lcl}
\hline Compound & Elemental analysis (\%) (theoretical) & \multicolumn{1}{c}{ FTIR spectral data $\left(\mathrm{cm}^{-1}\right)$} \\
\hline PTZ-CHL & $\mathrm{C}, 52 \cdot 41(52 \cdot 33)$ & $3384,3101,1631,1607,1529$, \\
$\left(\mathrm{C}_{18} \mathrm{H}_{9} \mathrm{NSCl}_{4} \mathrm{O}_{2}\right)$ & $\mathrm{H}, 2 \cdot 15(2 \cdot 20)$ & $1469,1430,1311,1278,1152,1106,1085$, \\
$(\mathrm{Mol}$. wt. $=413 \cdot 15)$ & $\mathrm{N}, 3 \cdot 31(3 \cdot 39)$ & $1034,918,879,855,741,705$ \\
& $\mathrm{~S}, 7 \cdot 83(7 \cdot 76)$ & \\
$\mathrm{PTZ}-\mathrm{PA}$ & $\mathrm{C}, 50 \cdot 59(50 \cdot 47)$ & $3383,3339,3101,1631,1603,1559$, \\
$\left(\mathrm{C}_{18} \mathrm{H}_{12} \mathrm{~N}_{4} \mathrm{O}_{7} \mathrm{~S}\right)$ & $\mathrm{H}, 2 \cdot 74(2 \cdot 82)$ & $1514,1530,1468,1442,1338,1314,1280$, \\
$(\mathrm{Mol}$. wt. $=428 \cdot 38)$ & $\mathrm{N}, 12 \cdot 99(13 \cdot 08)$ & $1252,1145,1080,1036,940,914,795,777$ \\
& $\mathrm{~S}, 7 \cdot 55(7 \cdot 48)$ & \\
\hline
\end{tabular}

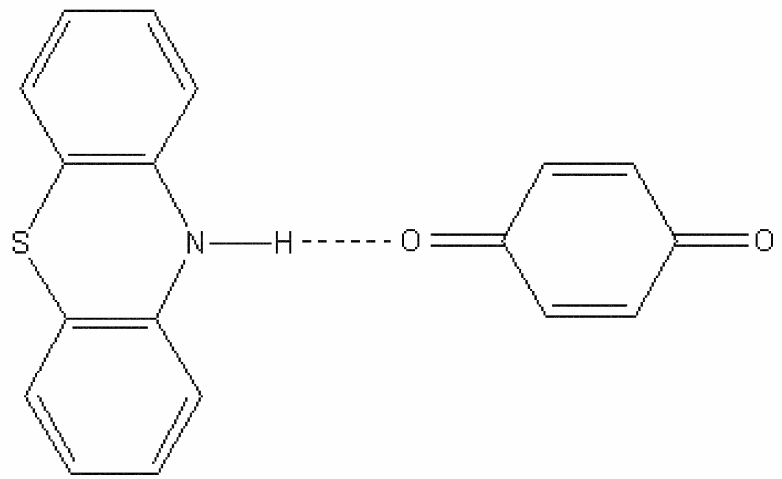

Figure 1. Phenothiazine-chloranil complex (PTZ-CHL).

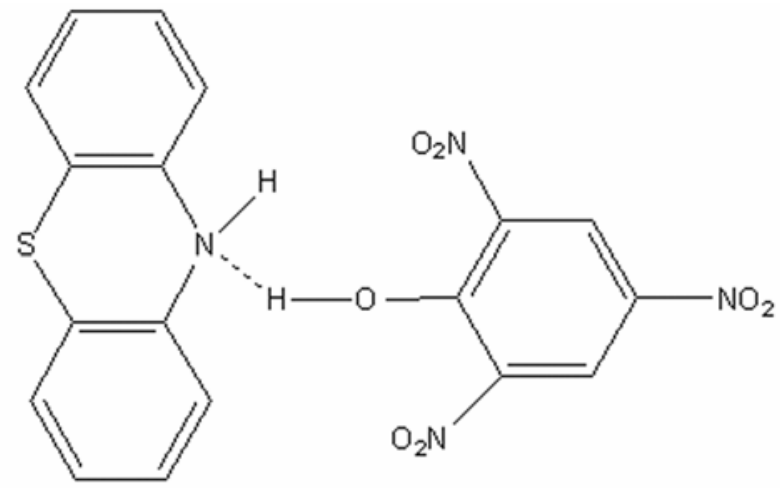

Figure 2. Phenothiazine-picric acid complex (PTZ-PA).

$\ln \left[\frac{1-y^{1-n}}{T^{2}(1-n)}\right]=\ln \left[\frac{A R}{\beta E_{\mathrm{a}}}\left(1-\frac{2 R T}{E_{\mathrm{a}}}\right)\right]-\frac{E_{\mathrm{a}}}{R T} \quad$ for $n \neq 1$.

Horowitz-Metzger:

$$
\begin{aligned}
& \ln [-\ln y]=\frac{E_{\mathrm{a}} \theta}{R\left(D T_{\max }\right)^{2}} \quad \text { for } n=1, \\
& \ln \left[\frac{1-y^{1-n}}{(1-n)}\right]=\frac{E_{\mathrm{a}} \theta}{R\left(D T_{\max }\right)^{2}} \quad \text { for } n \neq 1,
\end{aligned}
$$

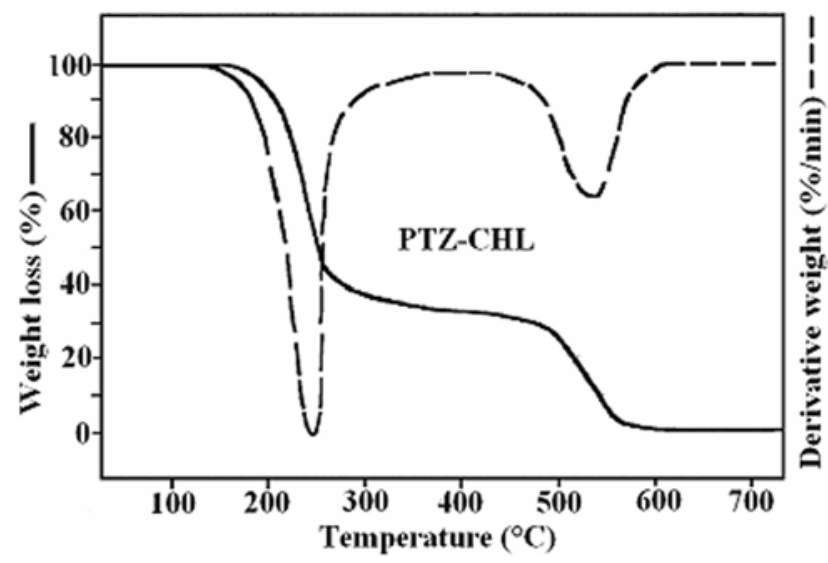

Figure 3. TGA (-) and DTG (---) thermograms of PTZCHL and PTZ-PA.

where $E_{\mathrm{a}}$ is the activation energy $(\mathrm{J} / \mathrm{mol}), R$ the universal gas constant $(8.314 \mathrm{~J} / \mathrm{mol}-\mathrm{k}), T$ the absolute temperature $\left({ }^{\circ} \mathrm{K}\right), D T_{\max }$ the maximum decomposition temperature, $A$ the Arrhenius pre-exponential factor $\left(\mathrm{s}^{-1}\right), n$ the reaction order, $\beta$ the heating rate $\left({ }^{\circ} \mathrm{C} / \mathrm{min}\right), \theta=T-D T_{\max }, y=\left(w_{\mathrm{t}}-w_{\infty} /\right.$ $\left.w_{0}-w_{\infty}\right)$, where $w_{0}, w_{\mathrm{t}}$ and $w_{\infty}$ are the weights of sample before degradation, at time, $t$ and after total decomposition, respectively. The graphical plots of $\ln [\ln (1 / y)]$ vs $1000 / T$ obtained for PTZ-CHL and PTZ-PA are presented in figure 4 . The value of ' $y$ ' presents the compound remaining at temperature, $T\left({ }^{\circ} \mathrm{K}\right)$. The slopes of the plots are determined and used to evaluate the activation energies. The values of ' $n$ ' reported in table 1 are the best fit values having the highest correlation coefficient. The activation energy observed are in the order PTZ-CHL > PTZ-PA. Greater the crystalline nature greater will be the activation energy (Mano et al 2003). The thermodynamic properties like change in enthalpy $(\Delta H)$, entropy $(\Delta S)$, free energy $(\Delta G)$ and frequency factor $(A)$ are calculated using standard equations as explained elsewhere (Daniels and Alberty 1955; Laidler 1972), which are summarized in table 2 . The first order rate constant is determined based on the weight changes with time in the linear degradation portion of the thermogravimetric curve and used for the evaluation of entropy change. The results obtained by the three methods are comparable except in a few cases. This is 
Table 2. A comparative, thermogravimetric analytical data of PTZ-CHL and PTZ-PA using Broido's, Coats-Redfern and Horowitz-Metzger methods

\begin{tabular}{|c|c|c|c|c|}
\hline & PTZ-CHL Step I & PTZ-CHL Step II & PTZ-PA Step I & PTZ-PA Step III \\
\hline \multicolumn{5}{|l|}{ Order $(n)$} \\
\hline Broido’s & 1 & 1 & 1 & 1 \\
\hline Coats-Redfern & $1 \cdot 0$ & $1 \cdot 0$ & $1 \cdot 0$ & $1 \cdot 0$ \\
\hline Horowitz-Metzger & $1 \cdot 0$ & $1 \cdot 0$ & $1 \cdot 0$ & $1 \cdot 0$ \\
\hline$D T_{\mathrm{m}}$ & 514 & 815 & 499 & 796 \\
\hline \multicolumn{5}{|l|}{$E_{\mathrm{a}}\left(\mathrm{kj} \mathrm{mol}^{-1}\right)$} \\
\hline Broido's & $87 \cdot 38$ & $69 \cdot 67$ & $44 \cdot 23$ & $83 \cdot 14$ \\
\hline Coats-Redfern & $72 \cdot 33$ & $57 \cdot 09$ & $33 \cdot 34$ & $68 \cdot 92$ \\
\hline Horowitz-Metzger & $80 \cdot 39$ & $72 \cdot 90$ & $38 \cdot 30$ & $82 \cdot 18$ \\
\hline \multicolumn{5}{|l|}{$\Delta H\left(\mathrm{kj} \mathrm{mol}{ }^{-1}\right)$} \\
\hline Broido’s & $83 \cdot 11$ & $62 \cdot 89$ & $40 \cdot 08$ & $76 \cdot 52$ \\
\hline Coats-Redfern & $68 \cdot 06$ & $50 \cdot 31$ & $29 \cdot 19$ & $62 \cdot 30$ \\
\hline Horowitz-Metzger & $76 \cdot 12$ & $66 \cdot 12$ & $34 \cdot 15$ & $75 \cdot 56$ \\
\hline \multicolumn{5}{|l|}{$\Delta G\left(\mathrm{kj} \mathrm{mol}{ }^{-1}\right)$} \\
\hline Broido's & $150 \cdot 77$ & $240 \cdot 93$ & $154 \cdot 30$ & $252 \cdot 69$ \\
\hline Coats-Redfern & $148 \cdot 11$ & $240 \cdot 61$ & $154 \cdot 39$ & $238 \cdot 36$ \\
\hline Horowitz-Metzger & $159 \cdot 92$ & $263 \cdot 56$ & 157.93 & $256 \cdot 20$ \\
\hline \multicolumn{5}{|l|}{$\Delta S\left(\mathrm{Jk}^{-1} \mathrm{~mol}^{-1}\right)$} \\
\hline Broido’s & $-131 \cdot 63$ & $-218 \cdot 45$ & $-229 \cdot 04$ & $-221 \cdot 32$ \\
\hline Coats-Redfern & $-155 \cdot 74$ & $-233 \cdot 50$ & $-250 \cdot 91$ & $-221 \cdot 18$ \\
\hline Horowitz-Metzger & $-163 \cdot 04$ & $-242 \cdot 26$ & $-248 \cdot 05$ & $-226 \cdot 93$ \\
\hline \multicolumn{5}{|l|}{$A\left(\mathrm{~s}^{-1}\right)$} \\
\hline Broido's & $14.3 \times 10^{3}$ & $66 \cdot 0$ & $11 \cdot 30$ & $45 \cdot 6$ \\
\hline Coats-Redfern & $78.6 \times 10^{3}$ & $10 \cdot 8$ & $8.1 \times 10^{-1}$ & $46 \cdot 4$ \\
\hline Horowitz-Metzger & $32.6 \times 10^{3}$ & $3 \cdot 8$ & $11.5 \times 10^{-1}$ & $23 \cdot 2$ \\
\hline
\end{tabular}

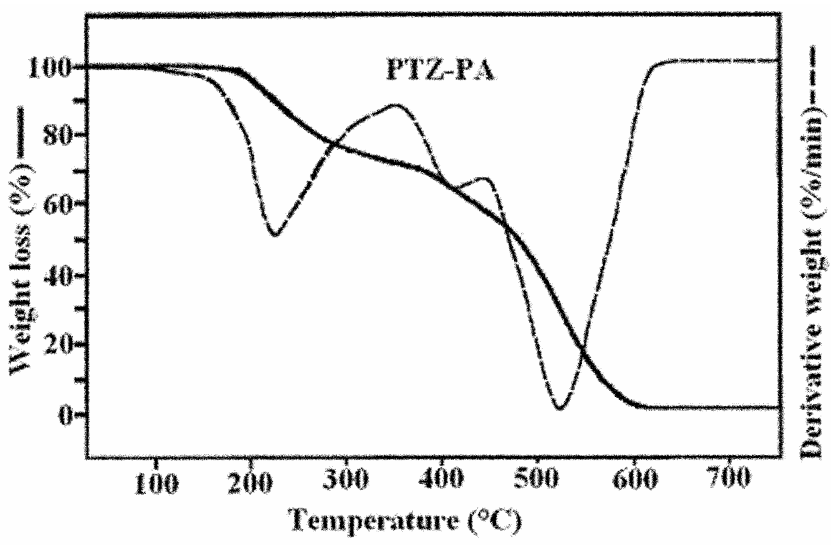

Figure 4. Graphical plots of $\ln [\ln (1 / y)]$ vs $1000 / T$ for PTZCHL and PTZ-PA.

because none of these methods are absolute ones with respect to mathematical approach as well as the assumptions.

\subsection{Electrical conductivity}

One of the best types of organic semiconductors is the C-T complex, which is formed by the interaction of electron donor with the electron acceptors. In general, in $\mathrm{C}-\mathrm{T}$ complexes, aromatic donors and acceptors stack, with the planes of the aromatic molecules parallel (Andrews and Keefer 1964). The repeat distance is usually that of the thickness of the two aromatic molecules (Wallwork 1961). Usually the primary electrical conduction is in the direction of the charge-transfer stack (Andrews 1954; Melby et al 1962; Gutmann and Lyons 1967). Most of the reported studies on electrical properties are based on polycrystalline materials compressed in the form of pellets due to difficulties in growing large single crystals. These polycrystalline materials have different grain sizes, grain boundaries etc. Electrical properties of $\mathrm{C}-\mathrm{T}$ materials depend largely on different types of packing (Haddon 1984). Segregated packing usually results in higher conductivities due to the smaller Coulomb barriers between different states of charges on similar molecules. The magnitude of the electrical conductivity of organic conductors is greatly affected by the arrangement of molecules in the crystal (Tanaka et al 1976; Bechgaard et al 1980). The conductivity measurements are carried out using the two-probe technique as explained in the experimental section on the powdered samples. In order to evaluate the nature of variations of electrical conductivity with temperature, electrical conductivity is measured from ambient to suitable high temperature. The higher tempe- 

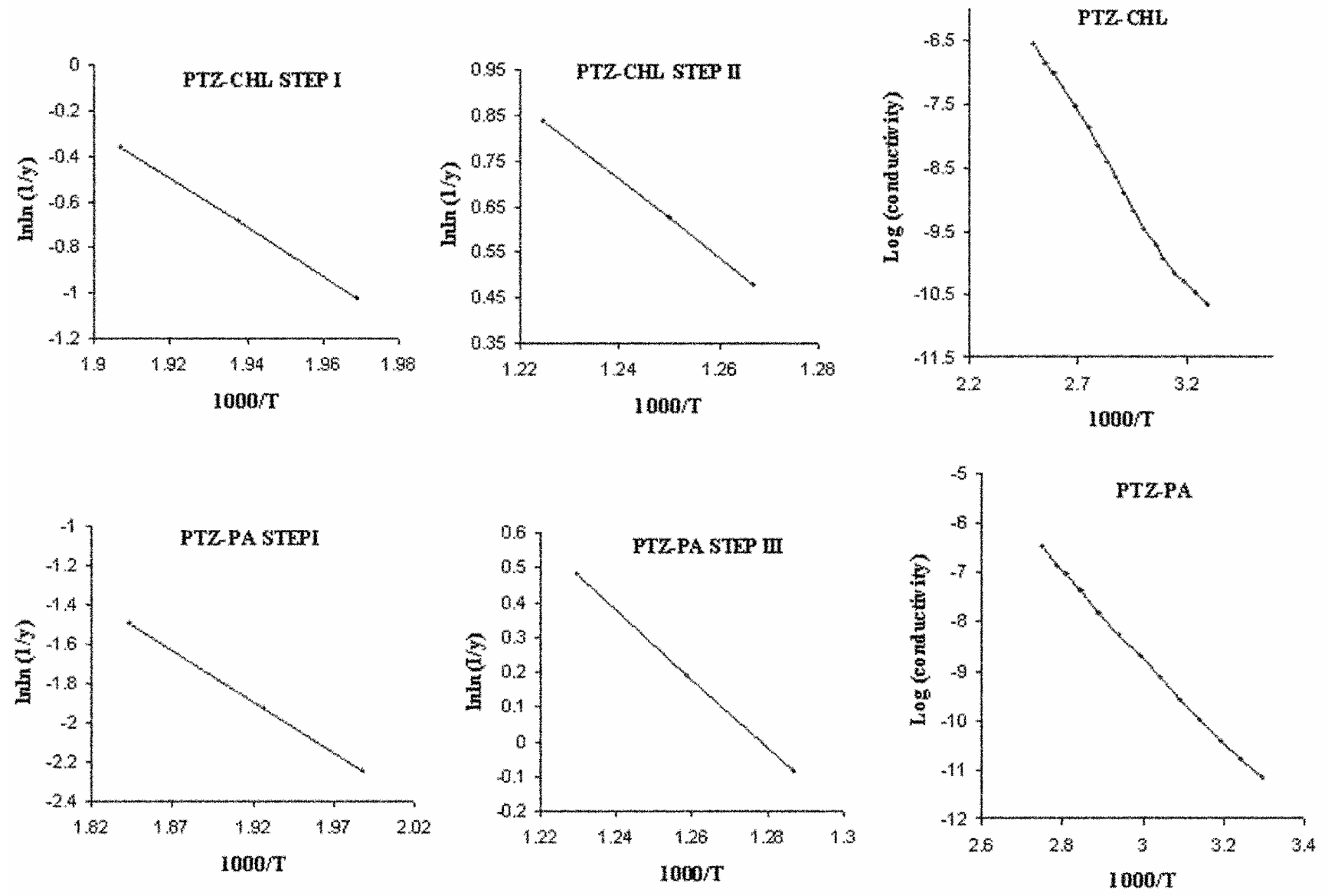

Figure 5. Electrical conductivity plots of PTZ-CHL and PTZ-PA.

rature limit selected for the electrical conductivity measurements are well within the melting point and decomposition temperatures of the phenothiazine $\mathrm{C}-\mathrm{T}$ complexes. The values of the electrical conductivity are calculated using the equation:

$$
\sigma=\sigma_{0} \exp ^{(-E / k T)}
$$

where $E$ is the activation energy, $k$ the Boltzmann constant, $T$ the temperature in ${ }^{\circ} \mathrm{K}$ and $\sigma_{0}$ a constant. The logarithmic conductivity values were plotted for each phenothiazine C-T complex vs 1000/T. Dependence of electrical conductivity of these complexes with temperature are shown in figure 5 and the relevant data are given in table 3. It has not been elucidated what rules determine the arrangement of molecules in the solid state, especially the formation of segregated stacks of molecules, though several workers have tried to define these rules (Saito and Ferraris 1980; Torrance 1987). The spatial orientation in phenothiazine molecule has a substantial effect on the geometry of charge-transfer compounds. When the hydrogen atom attached to nitrogen is replaced by a bulky alkyl group, it hinders the interaction between donor and acceptor. The net electrical conductivities observed for these phenothiazine $\mathrm{C}-\mathrm{T}$ complexes are due to the intra- molecular and inter-molecular electrical conductivities. The activation energy in organic semiconductors probably is a function of both intra- and intermolecular barriers, and therefore, is a composite of both. The structure of the molecule is of paramount importance as it may profoundly influence the electrical properties (Eley et al 1960). A few reports are available about the crystal structure of phenothiazine and its analogs (Feil et al 1965; Bell et al 1968; McDowell 1976; Waal and Feil 1976; Nakayama and Ishii 1987; Nakayama et al 1990). A cumulative opinion is that it exhibits temperature dependant structure. Crystal structures of a few phenothiazinium derivative picrates are reported elsewhere (Yathirajan et al 2007). Malrieu and Pullman (1964) suggested two extreme types of configuration for phenothiazine, viz. planar and the tetragonal folded one (Malrieu and Pullman 1964). In the folded tetragonal, $\mathrm{N}$ and $\mathrm{S}$ are in $S P^{3}$ hybridization state and the planes containing the benzene rings are folded along the axis passing through $\mathrm{N}$ and $\mathrm{S}$. The hydrogen atom attached to nitrogen can have two distinct configurations, called 'H-intra', with the hydrogen pointing inside and ' $\mathrm{H}$-extra', when the hydrogen atom points outside with respect to the dihedral angle. The two forms are not electronically equivalent. The 
Table 3. Electrical conductivity data of PTZ-CHL and PTZ-PA.

\begin{tabular}{lccc}
\hline \multirow{2}{*}{ Compound } & \multicolumn{2}{c}{ Conductivity $\left(\sigma \mathrm{S} \mathrm{cm}^{-1}\right)$} & \\
\cline { 2 - 3 } & & At maximum temperature & $E_{\mathrm{a}}(\mathrm{eV})$ \\
\hline PTZ-CHL & $3.62 \times 10^{-11}\left(30^{\circ} \mathrm{C}\right)$ & $2.69 \times 10^{-7}\left(110^{\circ} \mathrm{C}\right)$ & $0.54\left(30-95^{\circ} \mathrm{C}\right)$ \\
PTZ-PA & $2.05 \times 10^{-11}\left(30^{\circ} \mathrm{C}\right)$ & $3.30 \times 10^{-7}\left(90^{\circ} \mathrm{C}\right)$ & $0.75\left(45-80^{\circ} \mathrm{C}\right)$ \\
\hline
\end{tabular}

transition from intra to extra configuration leads to weakening of electron donor properties and to a decrease in energy of the upper bonding MO. Hydrogen bonding too has an effect on the activation energy and conduction mechanism (Rizk et al 1993). In several reported investigations high electrical conductivity obtained was due to hydrogen bonding (Pollock and Ubbelohde 1956; Brown and Aftergut 1963). Without crystal-structure results, more definite conclusions cannot be drawn regarding the effect of structure on the conduction mechanism for these $\mathrm{C}-\mathrm{T}$ complexes. Hence, experiments are underway and the findings would be communicated shortly.

\section{Conclusions}

The electrical conductivity studies on PTZ-CHL and PTZ-PA indicated that these C-T complexes showed semiconducting behaviour in the temperature range tested. The conductivities of both the complexes fall in the insulator range at $30^{\circ} \mathrm{C}$. Based on the thermogravimetric analysis in air, these complexes are thermally stable up to $90^{\circ} \mathrm{C}$. The data presented are useful for application in organic electronics. Conclusions concerning the nature of the charge carriers require more detailed investigations such as Hall effect, thermoelectric effect and carrier injection studies.

\section{Acknowledgement}

The authors would like to thank the Department of Science and Technology (DST), India, for funding the Vario EL-III elemental analyzer purchased under FIST programme.

\section{References}

Achar B N and Krishnaswamy V 1989 J. Electrochem. Soc. India 38311

Agarwal R K and Sivasubramanian M S 1987 AIChE. J. 337

Andrews L J 1954 Chem. Rev. 54714

Andrews L J and Keefer R M 1964 Molecular complex in organic chemistry (San Fransisco: Holden-Day) p. 55

ASTM Committee on Standards 1984 ASTM E698, Annual Book of ASTM Standards

Barigand M, Tondeur J J and Orszagh J 1970 B. Soc. Chim. Belg. 79625
Basu R and Choudhary M 1992 Spectrochim Acta A48 753

Bechgaard K, Jacobsen C S, Mortensen K, Pedersen H J and Thorup N 1980 Solid State Commun. 33119

Bell J D, Blount J F, Briscoe O V and Freeman H C 1968 Chem. Commun. 1656

Bordea C and Silberg I 1964 Rev. Roumaine Chim. 9505

Broido A 1969 J. Polym. Sci. 71761

Brown G P and Aftergut S 1963 J. Chem. Phys. 381356

Coats A W and Redfern J P 1964 Nature 20168

Daniels S F and Alberty R A 1955 Physical chemistry (New York: J. Wiley and Sons) p. 1

Darwish Ibrahim A 2005 Anal. Chim. Acta 549212

Dwivedi P C and Banga A K 1979 Electrochim. Acta 24831

Eley D D, Inokuchi H and Willis M R 1960 Disc. Faraday. Soc. 2854

Feil D, Linck M H and McDowell J J H 1965 Nature 2074994

Flynn J H and Wall L A 1966 J. Polym. Sci. Part B 4323

Freeman E S and Carrol B 1958 J. Phys. Chem. 62394

Friedman H L 1963 J. Polym. Sci. Part C 6183

Gangopadhyay J and Lahiri Sujit Chandra 2001 Chemie 215 883

Gutmann F and Keyzer H 1966 Electrochim. Acta 11 555, 1163

Gutmann F and Lyons L E 1967 Organic semiconductors (New York: Wiley) p. 462

Gutmann F, Hermann A M and Rembaum A 1967 J. Electrochem. Soc. 114323

Haddon R C 1984 Encyclopedia of semiconductor science and technology (ed.) M Grayson (New York)

Horowitz H H and Metzger G 1963 Anal. Chem. 351464

Iida Yoichi 1971 Bull. Chem. Soc. Jpn 44663

Jones G R N 1996 Med. Hypotheses 4625

Karpinska J, Starczewska B and Puzanowska-Tarasiewicz H 1996 Anal. Sci. 12161

Karremann G, Isenberg I and Szent-Gyoergi A 1959 Science 1301191

Kojlo A, Karpinska J, Kuzumicka L, Misink W, PuzanowskaTarasiewicz H and Tarasiewicz M 2001 J. Trace Microprobe T. 1945

Laidler K J 1972 Chemical kinetics (Tata McGraw Hill) 2nd edn, p. 1

Maher M A, Hamed E M Abdalla and Bayoumi Sh M 2003 Spectrosc. Letts 36357

Mano J F, Koniarova D and Reis R L 2003 J. Mater. Sci. Mater. Med. 14127

Malrieu J P and Pullman B 1964 Theor. Chim. Acta 293

Mattana A, Biancu G, Alberty L, Accardo A, Delogu G, Fiori P L and Cappuccinelli P 2004 Antimicrob. Agents Ch. 48 4520

McDowell J J H 1976 Acta Crystallogr. B32 5

Melby L R, Harder R J, Hertler W R, Mahler W, Benson R E and Mochel W E 1962 J. Am. Chem. Soc. 843374 
Nagy S, Argyelan G, Molnar J, Kawase M and Motohashi M 1996 Anticancer Res. 161915

Nakayama H and Ishii K 1987 Chem. Phys. 114431

Nakayama H, Mikako Mikai, Ryoji Hagiwara and Kikujiro Ishii 1990 J. Phys. Chem. 944343

Ordway D et al 2003 Antimicrob. Agents Ch. 47917

Ozawa T 1965 Bull. Chem. Soc. Jpn 381881

Pollock J M and Ubbelohde A R 1956 Trans. Faraday Soc. 52 1112

Rizk M S, Issa Y M, Ahmed M A and Shaaban S M 1993 J. Mater. Sci. 4109

Sadeghi S and Karimi E 2006 Chem. \& Pharm. Bull. 541107

Saito G and Ferraris J P 1980 Bull. Chem. Soc. Jpn 532141

Salem H 2002 J. Pharm. Biomed. Anal. 29527

Shi Ji-Liang, Zhou Cheng-Ming, Yi Hu-Nan, Qiu Zhi-Hai, Fu Yao-Hong and Jiang Xi-Kui 1998 Chin. J. Chem. 16397

Singh R A and Singh R 1996 Mol. Cryst. Liq. Cryst. 275195
Singh R A, Singh R, Rao O S and Verma S M 1993 Mol. Cryst. Liq. Cryst. 237419

Singh R and Singh R A 1997 Mol. Mater. 8187

Srivastava R D, Misra V S and Tripathi P N 1982 Electrochim. Acta 27863

Szocs G and Seiler N 2002 Acta Phytopathol. Hun. 37365

Tanaka J, Tanaka M, Kawai T, Takabe T and Maki O 1976 Bull. Chem. Soc. Jpn 492358

Tanaka M, Molnar J and Kidd S 1997 Anticancer Res. 17381

Torrance J B 1985 Mol. Cryst. Liq. Cryst. 55126

Torrance J B 1987 Low-dimensional conductors and superconductors (eds) D Jerome and L G Caron (New York: Plenum Publishing Corporation) p. 113

Van der Waal B W and Feil D 1976 Acta Crystallogr. B33 314

Wallwork S C 1961 J. Chem. Soc. 494

Yathirajan H S, Ashok M A, Narayana Achar B and Micheal Bolte 2007 Acta Crystallogr. E63 1432, 1691, 1693, 1792, 1795 